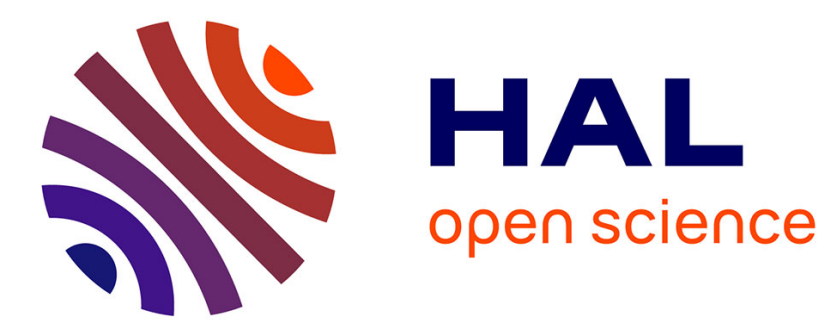

\title{
Superconducting point contacts weakly connecting two superconductors
}

\author{
R. de Bruyn Ouboter, A. Th. A. M. de Waele
}

\section{To cite this version:}

R. de Bruyn Ouboter, A. Th. A. M. de Waele. Superconducting point contacts weakly connecting two superconductors. Revue de Physique Appliquée, 1970, 5 (1), pp.25-31. 10.1051/rphysap:019700050102500 . jpa-00243369

\section{HAL Id: jpa-00243369 https://hal.science/jpa-00243369}

Submitted on 1 Jan 1970

HAL is a multi-disciplinary open access archive for the deposit and dissemination of scientific research documents, whether they are published or not. The documents may come from teaching and research institutions in France or abroad, or from public or private research centers.
L'archive ouverte pluridisciplinaire HAL, est destinée au dépôt et à la diffusion de documents scientifiques de niveau recherche, publiés ou non, émanant des établissements d'enseignement et de recherche français ou étrangers, des laboratoires publics ou privés. 


\title{
SUPERGONDUGTING POINT GONTAGTS WEAKLY GONNEGTING TWO SUPERGONDUGTORS
}

\author{
By R. DE BRUYN OUBOTER and A. Th. A. M. De WAELE,
} Kamerlingh Onnes Laboratorium der Rijksuniversiteit te Leiden, Leiden (Netherlands).

\begin{abstract}
The present review concerns dc and ac interference phenomena in superconducting point contacts weakly connecting two superconductors.

First, the properties of a single superconducting point contact will be discussed. Special attention will be paid to the resistive superconductive region, and its current-voltage characteristic. The point contact will be treated using a two-fluid model in which the total current is the sum of an ideal Josephson supercurrent $i_{s}(t)=i_{1} \sin \Delta \varphi^{*}(t)$ and a normal current $i_{n}=V / R_{n}=-(\hbar / 2 e)\left(\partial \Delta \varphi^{*}(t) / \partial t\right) / R_{n}\left(i_{1}\right.$ the maximum supercurrent ; $\Delta \varphi^{*}$ the gauge-invariant phase difference ; $V$ the voltage across the point contact and $R_{n}$ its normal resistance). It appears that small shunt capacitances and series inductances play an important role, even in its de current-voltage characteristic. Primarily, the two cases for which either the total current $i=i_{s}+i_{n}$ or the voltage $V$ are constants of time will be discussed. This approach appears to be essential for understanding the modulation in the voltage $\bar{V}\left(B_{\perp}\right)$ as a function of an external magnetic field at constant applied current $i$ for a double point contact.

Second, the external magnetic field dependence of the critical current (the dc Josephson effect) through a double point contact weakly coupling two superconductors is studied extensively, when the self-induced flux in the enclosed area is taken into account. Its relation with a strongly coupled double point contact, in which flux quantization holds, will be treated. Experimental results will be shown for the critical current of a symmetrical double point contact with such a small enclosed area $O=24 \times 10^{-8} \mathrm{~cm}^{2}\left(L=1.8 \times 10^{-12}\right.$ henry, $\left.i_{1}=185 \mu \mathrm{A}\right)$ that flux quantization is very incomplete : $\left.(h / 2 e) / L 2 i_{1} \approx 3>1\right)$ and will be compared with theory.

Finally, the properties of double point contacts in the resistive-superconductive region, in relation with both the ac and dc Josephson effects, will be discussed. It is observed that the dc voltage across a double point contact oscillates as a function of the applied magnetic field when a constant current is applied through the double point contact. The complete $\mathbf{i}-\mathbf{V}-B_{\perp}$ dependence can be represented by a corrugated surface. Two interpretations will be given : when the self-induced flux in the enclosed area is ignored and when it is taken into account respectively.

An extension is made by connecting two superconductors by more than two point contacts, which are adjusted on a straight line in such a way that an interference grating is formed.
\end{abstract}

The present review concerns dc and ac interference phenomena in superconducting point contacts weakly connecting two superconductors.

First, the properties of a single superconducting point contact $(\delta)$ weakly connecting two superconductors, with base-state wave functions $\Psi_{1}$ and $\Psi_{2}$, will be discussed (see fig. 1). The weak coupling is described by means of the Hamilton equations :

$$
i \hbar \frac{\partial \Psi_{k}(t)}{\partial t}=\sum_{l=1}^{2} H_{k l} \Psi_{l}(t)
$$

in which $H_{22}-H_{11}=2 \mathrm{eV} \quad(V=$ potential difference) and :

$$
\begin{aligned}
H_{21}=\langle 2|\hat{H}| 1\rangle_{A} \neq 0 \\
=\langle 2|\hat{H}| 1\rangle_{A=0} \exp \left(i \frac{2 e}{\hbar} \int_{1 \delta}^{2} A_{s} \mathrm{~d} s\right) \\
=K \exp \left(i \frac{2 e}{\hbar} \int_{1 \delta}^{2} A_{s} \mathrm{~d} s\right)=H_{12}^{*}
\end{aligned}
$$

(where $K=$ coupling constant in absence of a magnetic field). By substituting $\Psi_{k}=\sqrt{\rho_{k}} \exp (i \varphi) \quad(k=1,2$; $\rho_{k}=$ density of the superfluid particles), equating the real and imaginary parts of the Hamilton equations and taking $i_{s}=\dot{\rho}_{1}=-\dot{\rho}_{2}, \quad 2 K \sqrt{\rho_{1} \rho_{2}} / \hbar=i_{1}$, $\varphi_{2}-\varphi_{1} \equiv \Delta \varphi(t) \equiv$ the phase difference across the

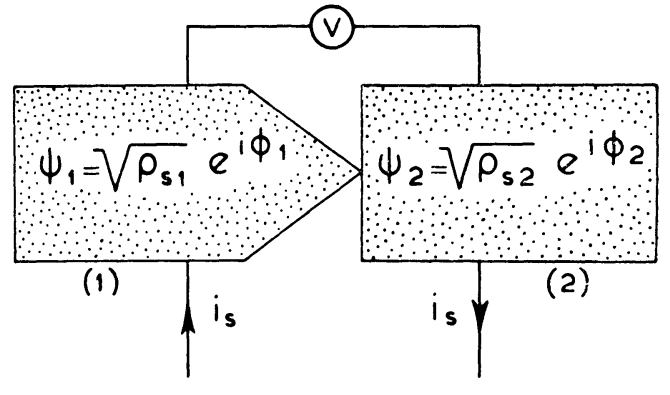

FIG. 1.

$$
i \hbar \frac{\partial \Psi_{k}}{\partial t}=\sum_{l=1}^{2} H_{k l} \Psi_{l}
$$

$$
\begin{gathered}
H_{21}=\langle 2|\hat{H}| 1\rangle_{\mathbf{A} \neq 0}=\langle 2|\hat{H}| 1\rangle_{\mathbf{A}=0} \mathrm{e}^{\frac{i \frac{2 e}{\hbar} \int_{\delta} A_{s} \mathrm{~d} s}{2}}=K \mathrm{e}^{i \frac{2 e}{\hbar} \int_{\delta} A_{s} \mathrm{~d} s}=H_{12}^{*} \\
\Delta \varphi \equiv \varphi_{2}-\varphi_{1} ; \quad i_{1} \equiv \frac{2 K}{\hbar} \sqrt{\rho_{1} \rho_{2}} \\
i_{s}(t)=i_{1} \sin \left(\Delta \varphi(t)-\frac{2 e}{\hbar} \int_{1 \delta}^{2} A_{s} \mathrm{~d} s\right) \equiv i_{1} \sin \Delta \varphi^{*}(t) \\
V=-\int_{1 \delta}^{2} E_{s} \mathrm{~d} s=-\frac{\hbar}{2} \frac{\partial \Delta \varphi^{*}}{\partial t} ; \quad \frac{\partial}{\partial t} \oint_{\text {voltmetercircuit }} A_{s} \mathrm{~d} s=0 .
\end{gathered}
$$


point contact, the Josephson relations for the supercurrent $i_{s}$ and the potential difference $V$ are found :

$$
\begin{gathered}
i_{s}(t)=i_{1} \sin \left(\Delta \varphi(t)-\frac{2 e}{\hbar} \int_{1 \delta}^{2} A_{s} \mathrm{~d} s\right)=i_{1} \sin \Delta \varphi^{*}(t) \\
V=-\frac{\hbar}{2 e} \frac{\partial \Delta \varphi}{\partial t}
\end{gathered}
$$

$\Delta \varphi^{*}$ is sometimes called the gauge-invariant phase difference. Mainly situations will be discussed in which the total current through the junction or the voltage over the junction are constant in time. If the total magnetic flux in the voltmeter circuit and in the current supply is constant in time $\left(\frac{\partial}{\partial t} \oint_{\text {circuit }} A_{s} \mathrm{~d} s=0\right)$ the measured voltage $V$ is equal to [1] :

$$
V=-\int_{1 \delta}^{2} E_{s} \mathrm{~d} s=-(\hbar / 2 e)\left(\partial \Delta \varphi^{*} / \partial t\right) .
$$

Equations (1) and (2) are quantitative formulations of the dc Josephson effect : a supercurrent $i_{s}=i_{1} \sin \Delta \varphi^{*}$ can flow through a point contact when no voltage difference $V$ is present and its maximum value $i_{1}$ is reached when $\Delta \varphi^{*}=\pi / 2$. Furthermore combining equations (1) and (2) gives a quantitative formulation of the ac Josephson effect : when a constant voltage difference is established across the point contact the supercurrent oscillates at a frequency $(2 e / h) V$ and with an amplitude $i_{1}$. If the point contact is brought into the resistive-superconductive region $(V(t) \neq 0)$, a normal current also has to be taken into account. A two-fluid model will be used in which the total current is the sum of an ideal Josephson supercurrent :

$$
i_{s}(t)=i_{1} \sin \Delta \varphi^{*}(t)
$$

and a normal current :

$$
i_{n}=V / R_{n}=-(\hbar / 2 e)\left(\partial \Delta \varphi^{*}(t) / \partial t\right) / R_{n}
$$

(see fig. 2).

The behaviour of a point contact is complicated because very small inductances and capacitances play an important role, even in its dc current-voltage characteristic. Stewart [2] and McCumber [3] calculated current-voltage characteristics of a point contact with a capacitance in parallel and also of a point contact with a self-inductance in series under different circuit conditions.

We will start with a somewhat different approach [1]. At first both the supercurrent and the normal current through the contact will be taken into account and we will ignore the self-inductance of the point contact and the capacitive coupling between the superconductors. However, we will apply certain limitations to the differential equations of the contact. Primarily the two cases for which either the current or the voltage are constants of time will be discussed.

We first take the voltage $V$ as a constant in time. If $V=0$, any dc supercurrent between $-i_{1}$ and $+i_{1}$ can flow through the junction. If $V \neq 0$, then the sinusoidal ac Josephson current oscillates at a frequency $\nu=(2 e / h) V$ and with an amplitude $i_{1}$. The time average of the supercurrent is equal to zero and only the normal component contributes to the dc current $\overline{i(t)}=i_{n}=V / R_{n} \quad($ see fig. $2 \mathrm{a})$. Voltage biasing can be achieved by shunting the junction with a parallel ideal resistance $R$ (when $i_{R} \gg i_{1}$ ) [4] or an ideal capacitance $C$ (with $\left.1 /(2 \pi \nu C) \ll R_{n}\right)[2]$, [3].

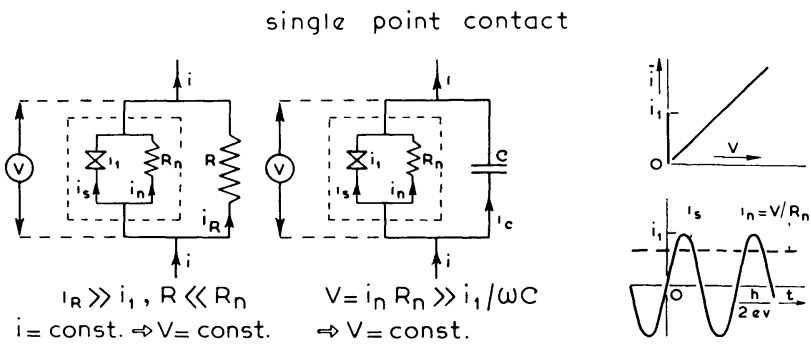

(a)
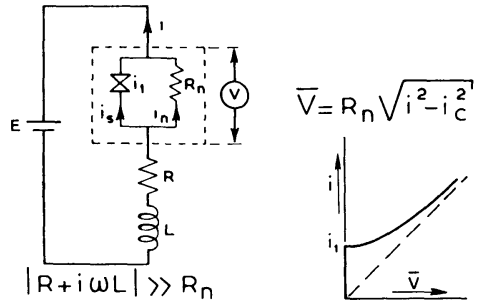

$i=i_{1} \sin \Delta \phi^{*}(t)-\frac{\hbar}{2 e} \frac{\partial \Delta \phi^{*}(t)}{\partial t} / R_{n}=$ const .
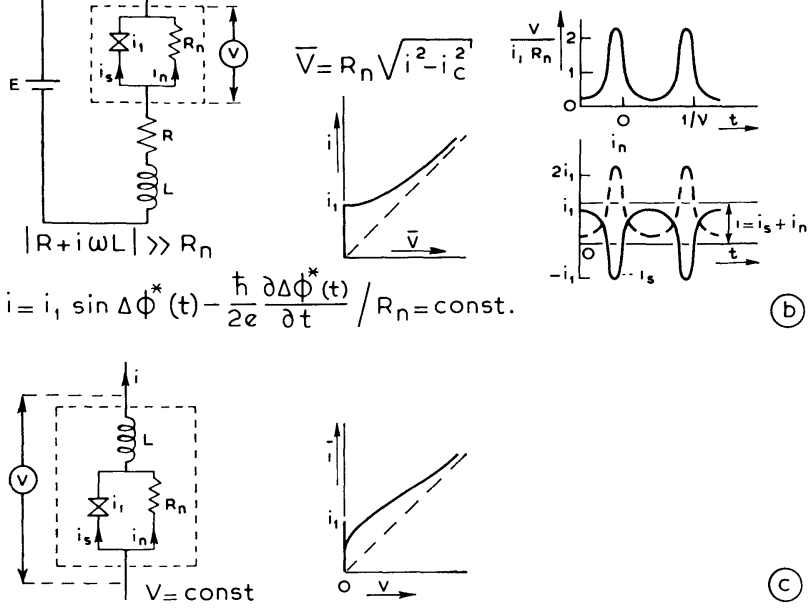

FIG. 2.

Second, we take the total current $i=i_{s}(t)+i_{n}(t)$ as a constant in time and calculate the mean voltage $\overline{V(t)}$. The solution of the differential equation :

$i=i_{s}+i_{n}=i_{1} \sin \Delta \varphi^{*}(t)-\frac{\hbar}{2 e}\left(\partial \Delta \varphi^{*} / \partial t\right) / R_{n}$

$=$ constant

gives the dc current $(i)$-voltage $\overline{(V(t)})$ characteristic :

$\overline{V(t)}^{t}=-\overline{(\hbar / 2 e)\left(\partial \Delta \varphi^{*} / \partial t\right)^{t}}=R_{n} \sqrt{i^{2}-i_{1}^{2}}$

which is given in figure $2 \mathrm{~b}$.

When $i>i_{1}$, then the voltage $V(t)$ is periodic and a sharply peaked function of time, especially when $i$ is only slightly larger than $i_{1}$ (see fig. $2 \mathrm{~b}$ ). The peakheight is equal to $2 i_{1} R_{n}$ and the width of the order $(h / 2 e) /\left(i_{1} R_{n}\right)$. The time dependence of the voltage $V(t)$, the normal current $i_{n}(t)=V(t) / R_{n}$ and the supercurrent $i_{s}(t)=i-i_{n}(t)$ are plotted in figure $2 \mathrm{~b}$. A constant current can be realised experimentally if one applies the current with a circuit having a high impedance [1], [3]. We like to remark, that this approach appears to be essential for understanding the observed modulation in the voltage $\overline{V\left(B_{\perp}\right)}$ as a function of an external magnetic field at constant applied current $i$ for a double point contact [1]. If one tries to measure the current-voltage characteristic with such a constant-current circuit, it is nearly impossible to avoid capacitive coupling between the two superconductors of order $10^{-10} \mathrm{~F}$. This capacitance plays a significant role for large values of $\overline{V(t)}$. When $\overline{V(t)}$ is increased the frequency of the ac currents increases and the capacitance becomes a short for ac currents between the superconductors $\left(R_{n} \gg 1 / 2 \pi \nu C\right)$. In this limit we can take $V(t)$ as a constant in time. We 
remark that McCumber [3] performed calculations on a point contact in series with a self-inductance while the system is voltage biased. Some results are shown in figure $2 \mathrm{c}$.

Second, the external magnetic field dependence of the critical current (the dc Josephson interference effect) through a double point contact weakly coupling two superconductors will be discussed extensively. The self-induced flux in the enclosed area is taken into account. The critical current $i_{\mathrm{c}}$ is defined as the maximum current that can flow through a double point contact without any voltage appearing across the junction. The critical current $i_{\mathrm{c}}\left(B_{\perp}\right)$ of a double point contact between two superconductors is a periodic function of the external magnetic field $B_{\perp}$, applied perpendicularly to the enclosed area $O$ between the contacts. This is a consequence of the fact that the phase differences
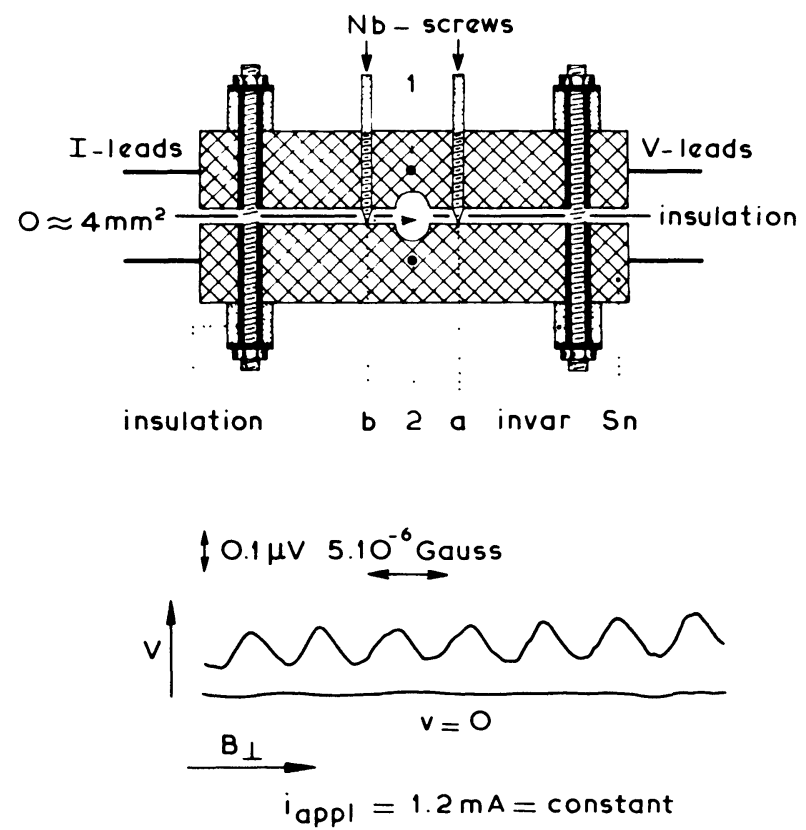

FIG. 3. - Weakly coupled double point contact :

$$
\begin{gathered}
\Delta \varphi_{a}=\left(\varphi_{2}-\varphi_{1}\right)_{a} ; \Delta \varphi_{b}=\left(\varphi_{2}-\varphi_{1}\right)_{b} \\
\oint \mathrm{d} \varphi=n 2 \pi=O=\Delta \varphi_{a}-\Delta \varphi_{b}+\frac{2 e}{\hbar} \oint_{\substack{\text { junction } \\
\text { excluded }}} A_{s} \mathrm{~d} s \\
\Delta \varphi_{b}^{*}-\Delta \varphi_{a}^{*}=\Delta \varphi_{b}-\Delta \varphi_{a}-\frac{2 e}{\hbar} \int_{1 b}^{2} A_{s} \mathrm{~d} s+\frac{2 e}{\hbar} \int_{1 a}^{2} A_{s} \mathrm{~d} s \\
=\frac{2 e}{\hbar} \oint_{s} A_{s} \mathrm{~d} s \\
\text { ju ction included } \\
i_{s}=i_{1}\left[\sin \Delta \varphi_{b}^{*}+\sin \Delta \varphi_{a}^{*}\right] \quad \\
=i_{1}\left[\sin \left\{\Delta \varphi_{a}^{*}+\frac{2 e}{\hbar} \oint A_{s} \mathrm{~d} s\right\}+\sin \Delta \varphi_{a}^{*}\right] \\
\oint A_{s} \mathrm{~d} s=B_{\perp} O+L i_{\text {circ }} \neq n \frac{h}{2 e} .
\end{gathered}
$$

across the two weak contacts are related to the embraced magnetic flux in the following way (see fig. 3) :

$$
\begin{aligned}
\oint_{s} \mathrm{~d} \varphi=n 2 \pi & =O=\Delta \varphi_{a}-\Delta \varphi_{b} \\
+ & \frac{2 e}{\hbar} \oint_{s}^{*} A_{s} \mathrm{~d} s\left(\oint_{s}^{*}=\text { junctions excluded }\right)
\end{aligned}
$$

in which :

$$
\Delta \varphi_{a} \equiv\left(\varphi_{2}-\varphi_{1}\right)_{a} \text { and } \Delta \varphi_{b} \equiv\left(\varphi_{2}-\varphi_{1}\right)_{b}
$$

are the phase differences across the weak contacts $a$ and $b$ respectively. The phase differences inside the bulk superconductors (1) and (2) were found by integrating the London eq. $\mathbf{p}_{s}=2 e \mathbf{A}=\hbar \nabla \varphi$ around the hole (junctions excluded). For the gauge-invariant phase difference $\Delta \varphi^{*}$, one obtains [1]:

$\Delta \varphi_{b}^{*}-\Delta \varphi_{a}^{*}=(2 e / \hbar) \oint_{s} A_{s} \mathrm{~d} s=(2 e / \hbar)\left(B_{\perp} O+L i_{\text {circ }}\right)$

$\left(\oint_{s}=\right.$ junctions included $)$, in which the total embraced magnetic flux consists of two parts : the applied magnetic flux $B_{\perp} O$ and a self-induced magnetic flux $\Phi_{s}=L i_{\text {circ }}$ ( $L=$ the self-inductance and $i_{\text {circ }}=$ the circulating current) (see fig. 3 or 4 ).

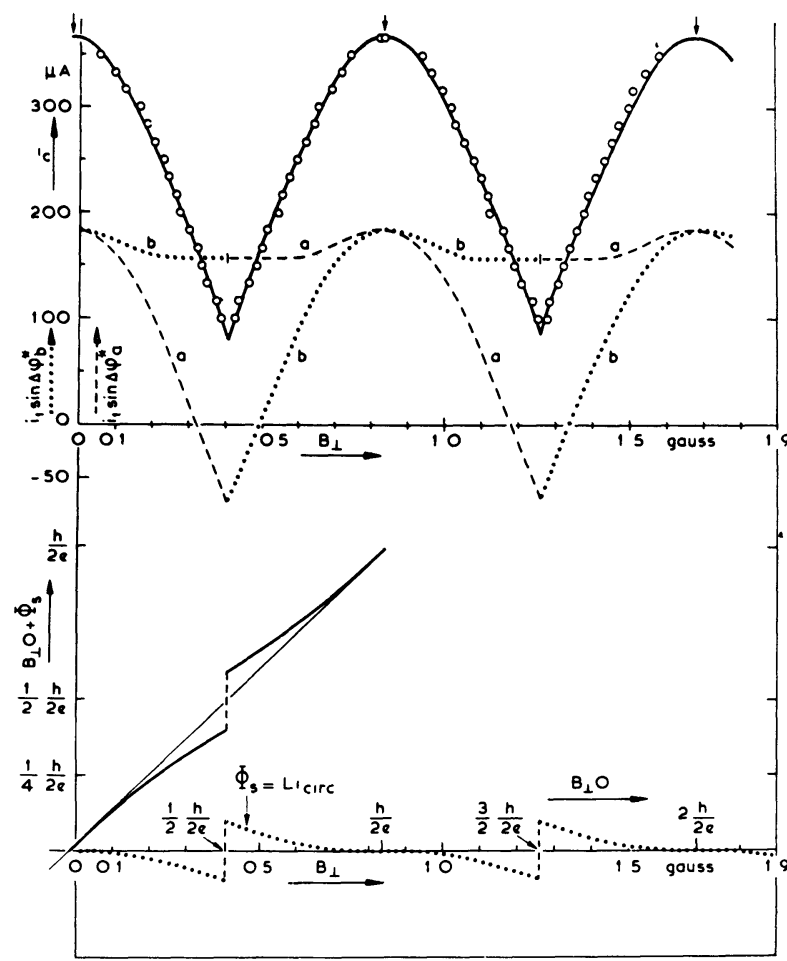

FIG. 4. - Weakly coupled symmetrical double point contact :

$$
\begin{gathered}
\frac{1}{2} i_{s}+i_{\text {circ }}=i_{1} \sin \Delta \varphi_{b}^{*} ; \quad \frac{1}{2} i_{s}-i_{\text {circ }}=i_{1} \sin \Delta \varphi_{a}^{*} \\
i_{s}=i_{1}\left[\sin \left\{\Delta \varphi_{a}^{*}+\frac{2 e}{\hbar}\left(B_{\perp} O+L i_{\text {circ }}\right)\right\}+\sin \Delta \varphi_{a}^{*}\right] \\
2 i_{\text {circ }}=i_{1}\left[\sin \left\{\Delta \varphi_{a}^{*}+\frac{2 e}{\hbar}\left(B_{\perp} O+L i_{\text {circ }}\right)\right\}-\sin \Delta \varphi_{a}^{*}\right] \\
i_{\max }=2 i_{1}=370 \mu \mathrm{A} \\
O=24 \times 10^{-8} \mathrm{~cm}^{2} \\
i_{\min }=84 \mu \mathrm{A} \\
\frac{h / 2 e}{2 i_{1} L} \approx 3>1 \quad L=0.86 \text { gauss }
\end{gathered}
$$

The total embraced magnetic flux is in general not equal to an integral number of magnetic flux quanta. When $i_{s}$ represents the externally applied supercurrent through a symmetrical double point contact (see fig. 4) then half the supercurrent $i_{s} / 2$ will flow through each contact. In addition to this there is the circulating supercurrent $i_{\text {circ }}$ associated with the self-inductance $L$ 
of the double contact. In one of the contacts both currents have the same direction, and in the other they are opposite. Hence the total supercurrent through each contact is equal to (see fig. 4) :

$$
i_{s} / 2+i_{\text {circ }}=i_{1} \sin \Delta \varphi_{a}^{*}
$$

and :

$$
i_{s} / 2-i_{\text {circ }}=i_{1} \sin \Delta \varphi_{b}^{*}
$$

respectively.

By addition and substraction one obtains :

$i_{s}=i_{1}\left[\sin \left\{\Delta \varphi_{a}^{*}+(2 e / \hbar)\left(B_{\perp} O+L i_{\text {circ }}\right)\right\}+\sin \Delta \varphi_{a}^{*}\right]$

$2 i_{\text {circ }}=i_{1}\left[\sin \left\{\Delta \varphi_{a}^{*}+(2 e / \hbar)\left(B_{\perp} O+L i_{\text {circ }}\right)\right\}\right.$

$$
\left.-\sin \Delta \varphi_{a}^{*}\right] \text {. }
$$

These two equations can be solved together by means of numerical or graphical methods. The critical current $i_{\mathrm{c}}$ is determined as the maximum of $i_{s}\left(B_{\perp}, \Delta \varphi_{a}^{*}\right)$ at a given magnetic field with respect to $\Delta \varphi_{a}^{*}$. The theoretical results obtained in this way can be verified experimentally.

In 1964-1965, Mercereau and his co-workers [5] constructed for the first time a quantum interferometer consisting of two parallel Josephson tunnel junctions (thin insulating oxide barriers) and Zimmerman and Silver [6] constructed interferometers in which the oxide tunnel junctions were replaced by superconducting point contacts. In order to measure the critical current-magnetic field dependence they used a method in which the time average of the voltage across the junction is measured when the positive part of a lowfrequency ac current, with amplitude larger than the maximum critical current, is applied on the junction. This complicated time average as a function of the magnetic field $B_{\perp}$ is more closely related to the complete current-voltage-magnetic field $\left(\bar{i}-\bar{V}-B_{\perp}\right)$ dependence [1], [7] (see later on) than to the real critical current-magnetic field $\left(i_{\mathrm{c}}-B_{\perp}\right)$ curve. Only a method determining $\lim _{\bar{V} \downarrow 0} i\left(\bar{V}, B_{\perp}\right)$ gives the critical current (if furthermore real current biasing in the point contact is applied).

Figure 4 gives one of our results for a symmetrical double point contact with a relatively small embraced area $0 \approx 24.10^{-8} \mathrm{~cm}^{-2}$ [1]. In this figure the experimental points are plotted together with the graph calculated from equations (6) and (7) using the values $(h / 2 e) /\left(L 2 i_{1}\right) \approx 3, i_{\mathrm{c}, \max }=2 i_{1}=370 \mu \mathrm{A}$ and $\Delta B_{\perp}$ $=0.86$ gauss. These values were chosen to give the best fit with the experimental results. We should like to point out that for this double contact having a small self-inductance, the maximum circulating current is considerable :

$$
i_{\text {circ }}=\left|i_{a}-i_{b}\right| / 2=0.62 i_{1}=115 \mu \mathrm{A}
$$

and the minimum critical current is finite :

$$
i_{\mathrm{c}, \min }=84 \mu \mathrm{A}
$$

at a half integral number of applied magnetic flux quanta $\left(B_{\perp} O=(n+1 / 2) h / 2 e\right)$. Although $i_{\text {circ }}$ is large the self-induced flux $\Phi_{s}=L i_{\text {circ }}$ is relatively small $(\approx(1 / 8) h / 2 e)$ compared with the value for pure flux quantization $((1 / 2) h / 2 e)$. On the other hand, at an integral number of applied magnetic flux quanta $\left(B_{\perp} O=n h / 2 e\right)$, the circulating current is equal to zero. In the upper part of the figure the experimental points for the critical current-magnetic field curve are plotted. The lines representing the currents through the individual contacts $(a)$ and $(b)$ and the total critical current $(a+b)$ have been calculated [1]. Shown in the lower part of the figure are graphs of the selfinduced flux $\Phi_{s}=L i_{\text {circ }}$ and of the total embraced flux $B_{\perp} O+\Phi_{s}$, both calculated for the case where the junction is critical. For the value :

$$
(h / 2 e) /\left(L 2 i_{1}\right) \approx 3>1
$$

the flux quantization is very incomplete.

When a half integral number of flux quanta is applied on $O$, the critical current is a minimum $\left(i_{\mathrm{c}}=i_{\mathrm{c}, \min }\right)$. This critical current is shown in figure 5 as a function of $\pi L i_{1} /(h / 2 e)$. The currents through the individual

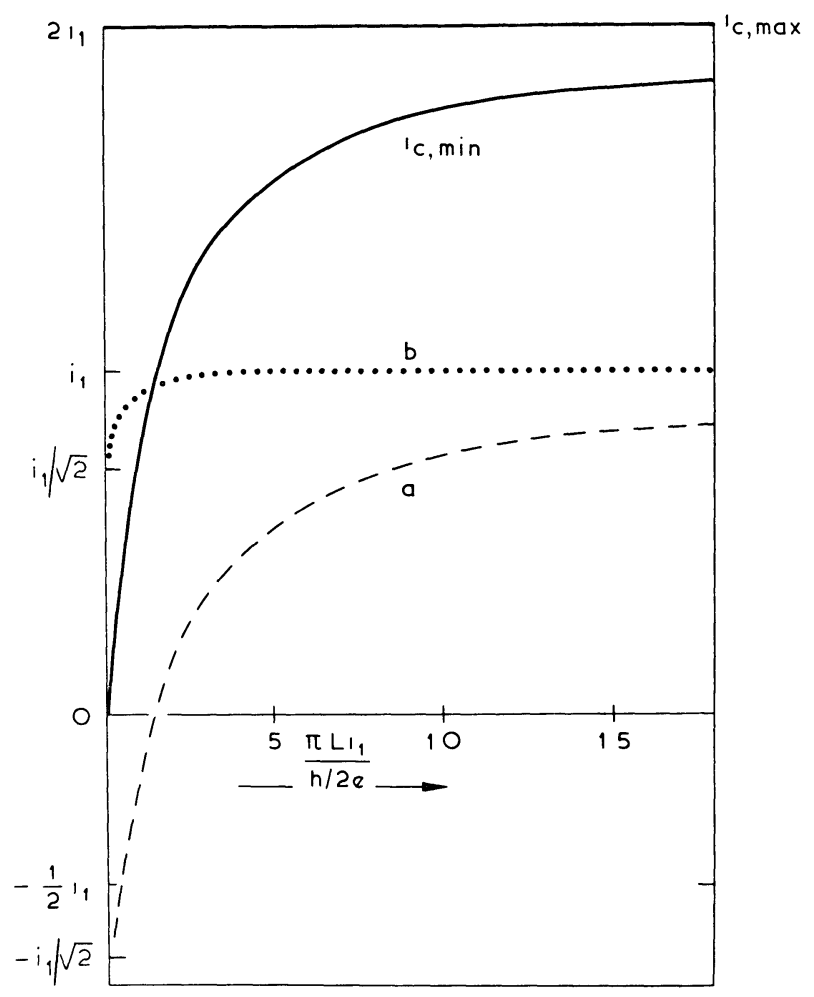

FIG. 5.

contacts are also shown. When $\pi L i_{1} /(h / 2 e) \rightarrow 0$ the minimum critical current is equal to zero $\left(i_{a}+i_{b}=0\right)$ and the circulating current equal to $\left(i_{a}-i_{b}\right) / 2=i_{1} / \sqrt{ } \overline{2}$. When $\pi L i_{1} /(h / 2 e) \gg 1$, the equations (6) and (7) show that the total magnetic flux in the hole is nearly quantized in units $h / 2 e$.

Far away to the right of this figure lays the main interest of this conference : the detection of very weak magnetic fields. The amplitude of the modulation in the critical current is in this limit equal to $1 / 2(h / 2 e) / L$, as is derived and shown in figure 6 [1]. The total magnetic flux is quantized : $B_{\perp} O+L i_{\text {circ }}=n(h / 2 e)$. Due to flux conservation the applied current $i_{s}$ divides equally between both contacts of the symmetrical double junction. The circulating current adds to the applied current in one of the contacts $(a)$ and subtracts from the other $(b)$. When the applied current is increased from zero the critical current is reached when the total current through one of the contacts $(a)$, in which the applied current and the circulating 
current have the same direction, reaches its critical value $i_{1}$. Hence $i_{\mathrm{c}} / 2+i_{\text {circ }}=i_{1}$ or :

$i=i_{\mathrm{c}}=2 i_{1}-2\left|i_{\mathrm{circ}}\right|=2 i_{1}-\frac{2}{L}\left|n(h / 2 e)-B_{\perp} O\right|$

(see fig. 6).

Finally, the properties of double point contacts in the resistive-superconductive region, in relation with both the ac and dc Josephson effects, will be discussed. It is observed [1], [7] that the dc voltage across a double point contact oscillates as a function of the applied magnetic field when a constant current is applied through the double point contact. Some typical results are shown in figure 6 . The complete $\bar{i}-\bar{V}-B_{\perp}$ dependence

$\Phi=B_{\perp} O+L i$ circ $=n \frac{h}{2 e}$
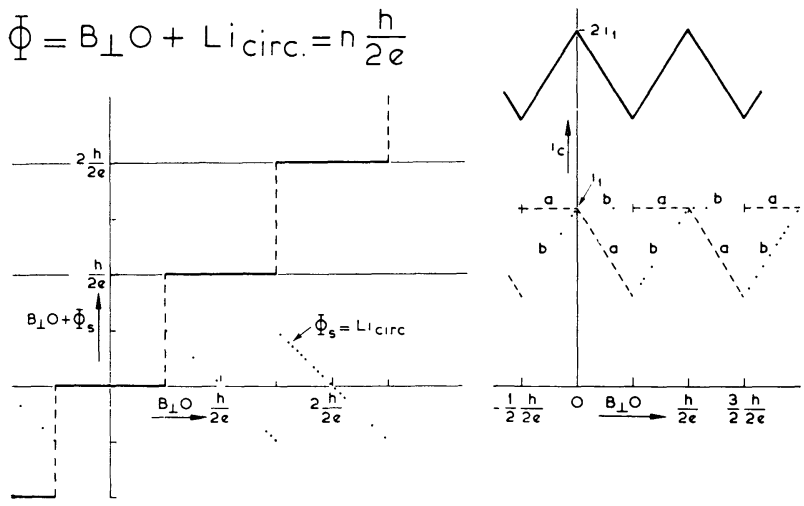

FIG. 6. - "Strongly" coupled double junction :

$$
i_{\mathrm{c}}=2 i_{1}-2 i_{\text {circ }}=2 i_{1}-\underbrace{\frac{2}{L}\left|n \frac{h}{2 e}-B_{\perp} O\right|}_{\max =\frac{h}{2 e} / L}
$$

can be represented by a corrugated surface [7]. The critical current $i_{\mathrm{c}}$ and the dc voltage $\bar{V}$ (at constant applied current) are both periodic functions of the applied magnetic field (with a period $\Delta B_{\perp}=(h / 2 e) / O$ ). A maximum in the critical current corresponds to a minimum in the voltage. The amplitude of both the $i_{\mathrm{c}}$ and $V$ oscillations decrease when the enclosed area $O$ between the contacts is increased. Double point contacts with a small enclosed area $\left(O \approx 60 \mu^{2}\right.$, $L \approx 10^{-12}-10^{-11}$ henry) can be obtained by simply pressing a superconducting point on a superconducting sheet or by using a solder-droplet junction ( $f i g .7 \mathrm{a}$ and $\mathrm{b})$. On the other hand the largest area in which oscillations were measured was $\approx 0.75 \mathrm{~cm}^{2}$, $L \approx 10^{-8}$ henry [6] (see also fig. 3).

In double point contacts ac properties can be observed as in single point contacts. The electromagnetic radiation emitted by the point contacts interferes. One can control the phase difference of the radiation emitted by the two contacts by means of an applied magnetic field [8]. The behaviour of a double point contact with a small enclosed area in its own resonant electromagnetic radiation field can be observed by putting it in the center of a microwave cavity. This is an extention of a technique due to Dayem and Grimes [9] (see fig. 7 below). We observed [8] that the current-voltage curve shows steps at constant voltages, corresponding to a resonant frequency of the
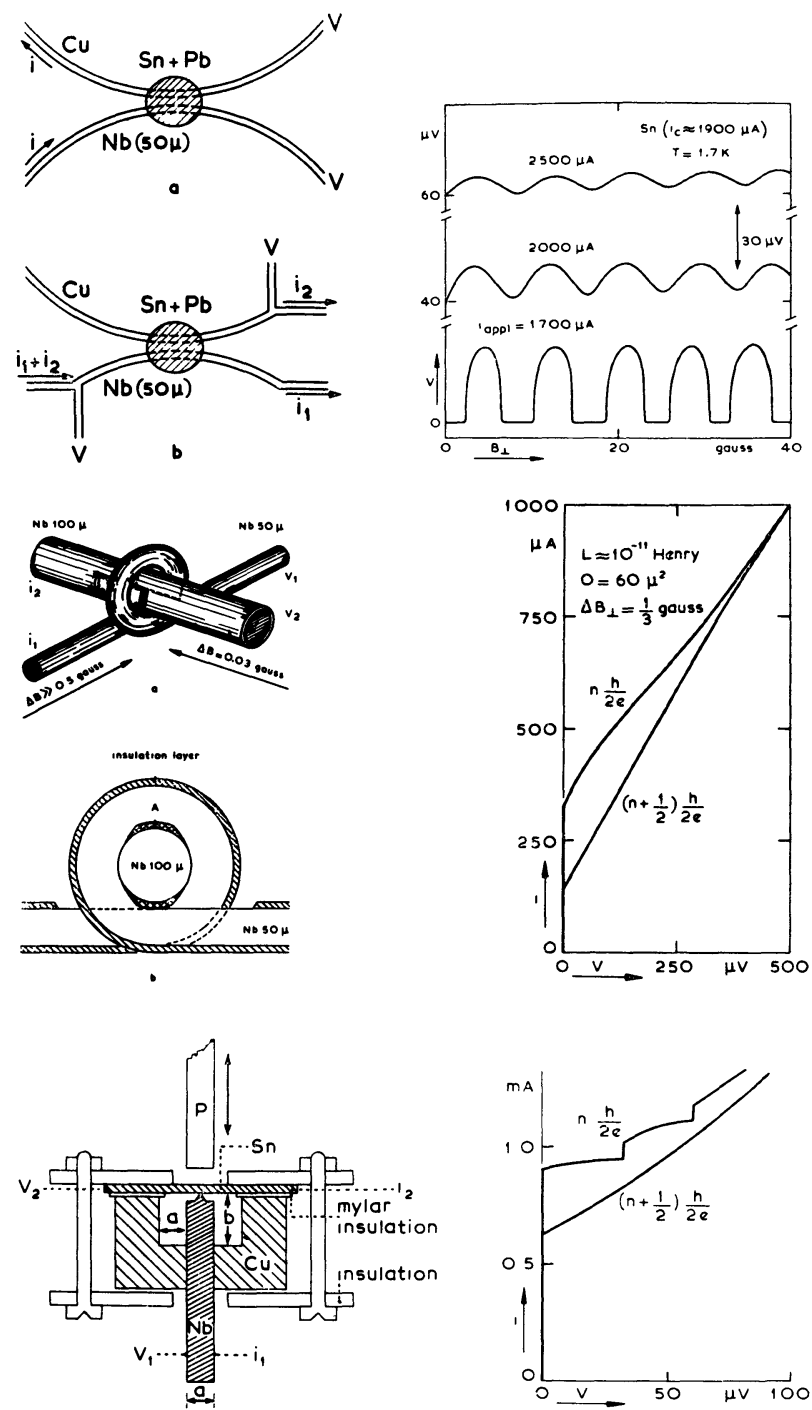

FIG. 7.

cavity, which were most pronounced when the applied flux on $O$ was equal to an integral number of flux quanta $\left(B_{\perp} O=n h / 2 e\right)$ and disappeared when the applied flux was equal to a half integral number of flux quanta $\left(B_{\perp} O=(n+1 / 2) h / 2 e\right)$.

Explanation: The total current through the junction is equal to the sum of the supercurrents through the individual contacts and the normal current. Again the total current is taken as a constant in time (see fig. $8 \mathrm{~b})$ :

$$
\begin{array}{r}
i=i_{1}\left[\sin \left\{\Delta \varphi_{a}^{*}+\frac{2 e}{\hbar}\left(B_{\perp} O+L i_{\text {circ }}\right)\right\}+\sin \Delta \varphi_{a}^{*}\right] \\
-\frac{\hbar}{2 e}\left(\partial \Delta \varphi^{*} / \partial t\right) / R_{n}=\text { constant }
\end{array}
$$

For simplicity, we start by ignoring the self-induced flux $\left(\Phi_{s}=L i_{\text {circ }}=0\right)$ in the enclosed area $O$, which 


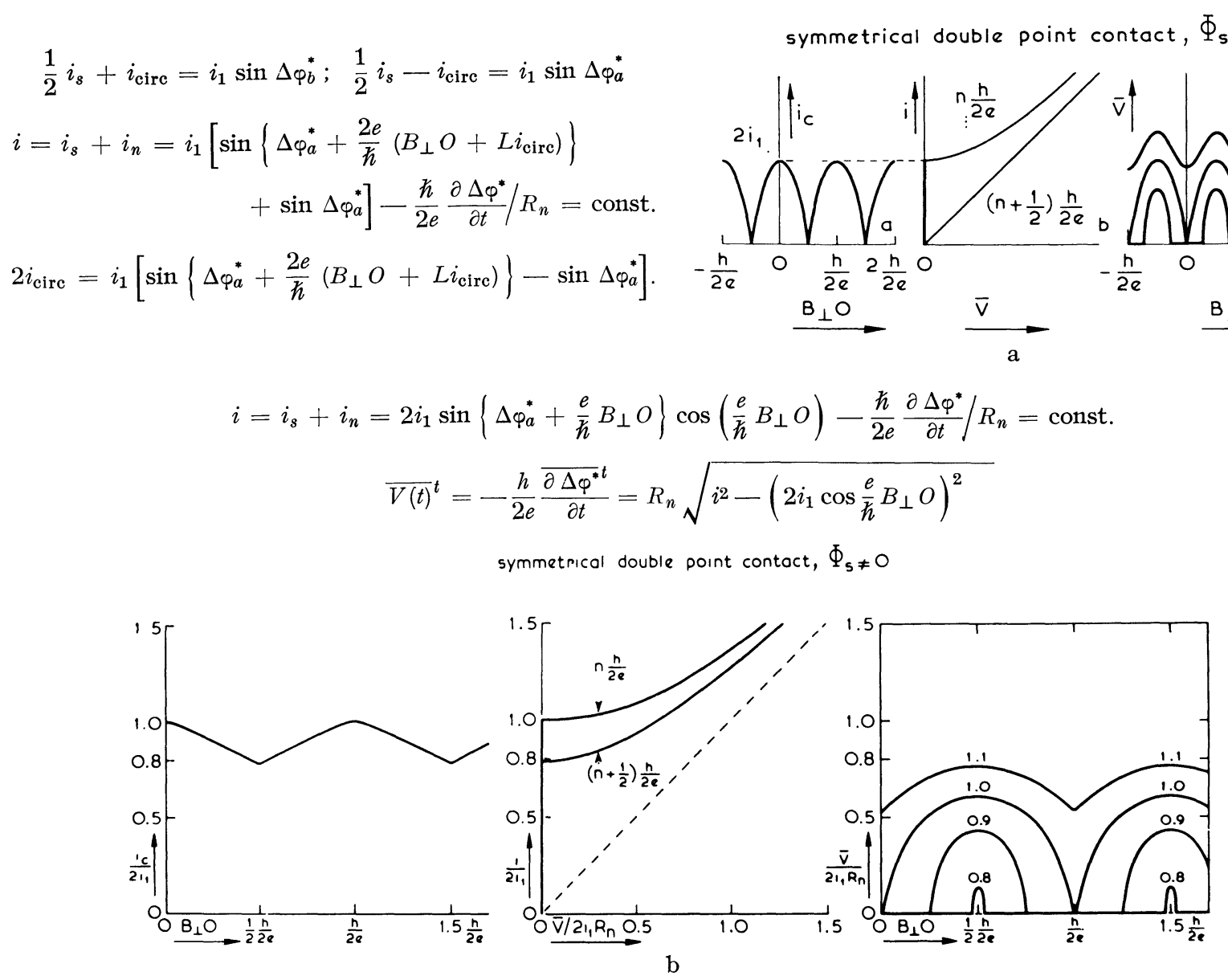

FIG. 8.

has the advantage that a simple analytical solution can be obtained. Equation (9) reduces to :

$i=2 i_{1} \sin \left(\Delta \varphi_{a}^{*}(t)+\frac{e}{\hbar} B_{\perp} O\right) \cos \left(\frac{e}{\hbar} B_{\perp} O\right)$

$$
-(\hbar / 2 e)\left(\partial \Delta \varphi^{*} / \partial t\right) / R_{n}=\text { constant }
$$

(see fig. $8 \mathrm{a}$ ), which is essentially the same as equation (3) when $i_{1}$ is replaced by $i_{\mathrm{c}}=2 i_{1}\left|\cos \frac{e}{\hbar} B_{\perp} O\right|$. Hence the solutions of the equations are the same : $\overline{V(t)}^{t}=-(\hbar / 2 e){\overline{\left(\partial \Delta \varphi^{*} / \partial t\right)}}^{t}$

$$
=R_{n} \sqrt{i^{2}-\left\{2 i_{1} \cos \left(\frac{e}{\hbar} B_{\perp} O\right)\right\}^{2}} \text {. }
$$

This result already explains the observed voltage oscillations in the resistive-superconductive region as a function of the external magnetic field $B_{\perp}$, for the case that the applied current $(i)$ is constant and exceeds the critical value $\left(i>i_{\mathrm{c}}\left(B_{\perp}\right)\right)$. The current-voltage characteristic $(i-\bar{V})$ for $i_{\mathrm{c}}=2 i_{1}$ (when $\left.B_{\perp} O=n h / 2 e\right)$ and for $i_{\mathrm{c}}=0$ (when $B_{\perp} O=(n+1 / \overline{2}) h / 2 e$ ) are represented in figure $8 \mathrm{a}$. In the same figure, we have also shown the voltage oscillations $\bar{V}$ as a function of the applied magnetic flux $B_{\perp} O$ at several values of a constant applied current.

If we drop the condition that the self-induced flux in the hole is equal to zero $\left(\Phi_{s}=L i_{\text {circ }} \neq 0\right)$ it is no longer possible to give a simple analytical solution of equations (9) and (10). The results obtained from

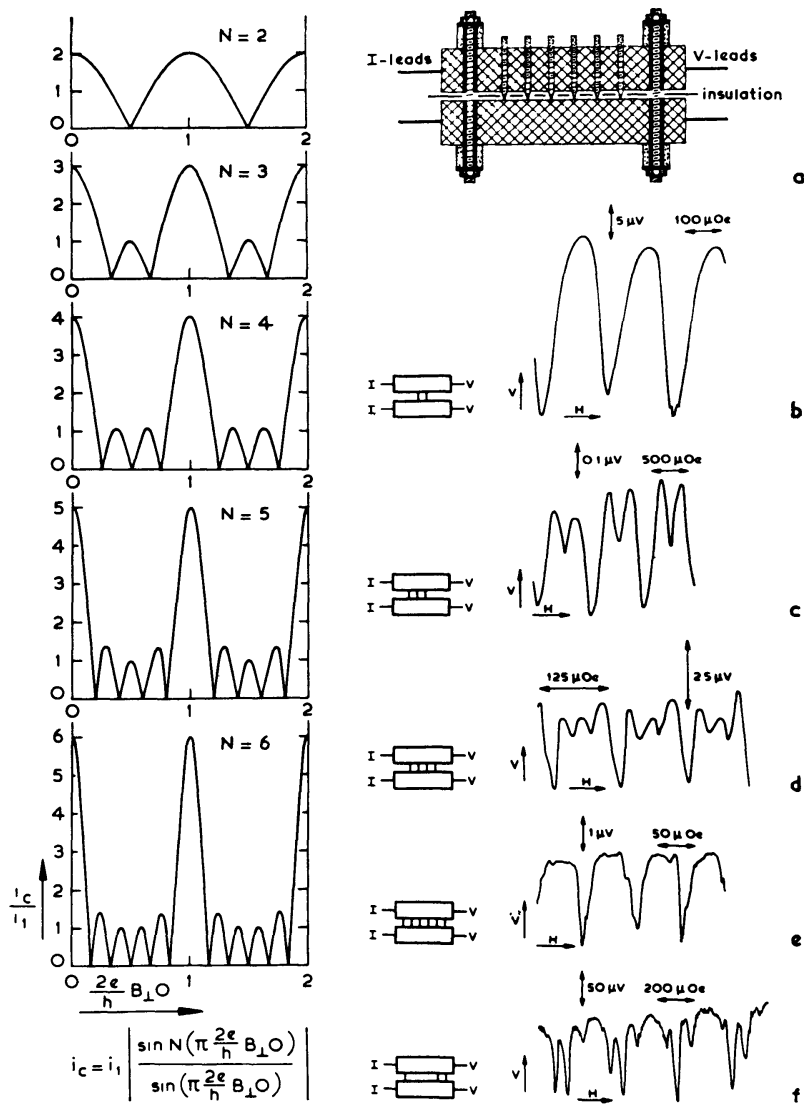

FIG. 9. 
computer calculations solving these equations (9) and (10) are shown in figure $8 \mathrm{~b}$ [1]. From left to right :

a) The critical-current oscillations $i_{\mathrm{c}}\left(B_{\perp}\right)$;

b) Two current-voltage characteristics when $B_{\perp} O$ $=n h / 2 e$ and when $B_{\perp} O=(n+1 / 2)(h / 2 e)$;

c) The voltage oscillations as a function of the applied magnetic flux $B_{\perp} O$ at several values of a constant applied current.

A logic extension of the discussions given above can be made by connecting two superconductors by more than two point contacts, which are adjusted on a straight line in such a way that an interference grating is formed [10], [6], as is shown in figure 9. The left series of graphs give the calculated critical current as a function of the magnetic field for gratings of 2 to 6 weak contacts ignoring self-inductances and mutual inductances and assuming all the contacts are identical [10], [1]. The right-hand side of figure 9 gives the observed voltage oscillations as a function of the applied magnetic field for various assemblies. The type of assembly is indicated at the left of each experimental curve.

\section{REFERENCES}

[1] DE BRUYN OUBOTER (R.) and DE WAELE (A. Th. A. M.), Superconducting point contacts weakly connecting two superconductors, to be published in Progress in Low Temperature Physics, volume VI (1969), ed. by GORTER (C. J.), NorthHolland Publishing Company, Amsterdam; see also Physica, 1969, 41, 225, and 1969, 42, 626 .

[2] Stewart (W. C.), Appl. Phys. Letters, 1968, 12, 277.

[3] MCCumber (D. E.), J. Appl. Phys., 1968, 39, 297 ; 1968, 39, $2503 ; 1968,39,3113$.

[4] Zimmerman (J. E.), Cowen (J. A.) and Sillver (A. H.), Appl. Phys. Letters, 1966, 9, 353.
[5] Jakifivic (R. C.), Lambe (J.), Mercereau (J. E.) and Silver (A. H.), Phys. Rev., 1965, 140, A 1628 ; Phys. Rev. Letters, 1964, 12, 159 ; 1964, 12, 274 ; 1965, 14, 887.

[6] Zimmerman (J. E.) and Silver (A. H.), Phys. Letters, 1964, 10, 47 ; Phys. Rev., 1966, 141, 367.

[7] OMAR (M. H.), KraAN (W. H.), DE WAELE (A. Th. A. M.) and DE BRUYN OUBOTER (R.), Physica, 1967, 34, 525 ; Physica, 1966, 32, 1448.

[8] DE BRUYN OUboter (R.), KRAAN (W. H.), DE WAEle (A. Th. A. M.) and OMaR (M. H.), Physica, 1967, 37, 114.

[9] Dayem (A. H.) and Grimes (C. C.), Appl. Phys. Letters, 1966, 9, 47.

[10] DE WaEle (A. Th. A. M.), KraAN (W. H.) and DE Bruyn Ouboter (R.), Physica, 1968, 40, 302. 